\title{
Co-optimizing High and Low Voltage Systems: Bi-Level vs. Single-Level Approach
}

\author{
Jialin Liu \\ Cornell University \\ j13455cornell.edu
}

\author{
Luckny Zéphyr \\ Laurentian University \\ lzephyr@laurentian.ca
}

\author{
Judith Cardell \\ Smith College \\ jcardell@smith.edu
}

\begin{abstract}
This paper presents a bi-level optimization framework applied to optimize system performance with (i) increasing presence of distributed energy resources (DER) at the low-voltage level, and (ii) variable wind power generation at the high-voltage level. The paper investigates various system configurations with increasing presence of microgrids, with active devices. System simulations quantify system performance in terms of cost, first using the traditional single-level optimization framework, and second using the proposed bi-level framework. Comparisons between the system with traditional, passive distribution systems and with microgrids are also presented, with results again quantified via the interconnected system operating costs. Results show that at low levels of DER and microgrid penetration, traditional (single-level) system optimization algorithms perform adequately as compared to the proposed bi-level optimization framework. However, as DER and microgrid penetration increase, the traditional single-level framework does not accurately capture the full system benefits of distributed technologies. The results demonstrate that new optimization algorithms, such as the proposed bi-level framework, will be required if the benefits of DER are to be accurately quantified in the evolving power system.
\end{abstract}

\section{Introduction}

The interconnected power system was originally developed with centralized power generating facilities connected to a meshed high voltage network that delivered power to customers predominantly connected via passive, radial, distribution systems. A gradual deregulation and restructuring of the electric power industry began in the 1970 s and continues at a measured pace today [1]. This process has facilitated the inclusion of alternate distributed energy resources (DER) such as small-scale distributed and renewable generation, flexible demand technologies, along with moderate autonomy for independent power producers [2]. These changes challenge the centralized system control paradigm of the legacy power system.

Distributed and smart energy resources have been poised to transform the power system for decades. Costs for renewable energy steadily decrease. Customers' desire to limit negative impacts of power generation and transmission increases. A wary fascination of electric vehicles as well as home automation is ever present. What then explains the continued dominance of centralized generating facilities and passive customer engagement in the power industry? The causes of slow system evolution are numerous and often well-justified. Nonetheless, society as a whole will benefit from DER and smart technology penetration expanding as rapidly as possible.

Equally important to this evolution of physical devices on the power system are the software algorithms and protocols used to analyze and operate the system. The historical operating strategies assumed the historical power system structure, e.g., active and highly meshed central generating facilities serving customers located in passive, radial distribution systems. In this system configuration, high resolution energy data from distribution systems was neither available nor necessary for reliable system operation. The current direction for system evolution promotes a significant increase in both distributed and renewable energy generating technologies. Advances in automation, personal area networking and communications technologies facilitate both increased flexible load across end-use devices as well as two-way communication of high resolution energy usage and system state data from customers and low voltage systems to the grid operators. Faced with this mixture of legacy system elements and newer, advanced technologies, algorithms for system analysis and operations need to evolve to fully exploit the benefits of, and also avoid potential negative impacts of new DER and renewable energy technologies.

With this view of the existing and near-future 
power system, this paper investigates the use of bi-level co-optimization algorithms for system analysis. Specifically, at what level of DER penetration does it become important to use the proposed optimization algorithms in order to fully capture the expected benefits of DER to system performance. Results compare the simulated system performance as determined by single-level, traditional optimization to that determined by the proposed bi-level optimization that incorporates detailed knowledge of the distribution system as a microgrid with multiple DER installed.

Section 2 introduces the bi-level optimization modeling framework, Section 3 discusses results and Section 4 concludes. Nomenclature is included as an appendix.

\section{Bi-Level Modeling Framework}

In the proposed bi-level framework, the upper level optimization problem represents the transmission system unit commitment while the lower level is an optimal power flow that includes the microgrid elements. This bi-level framework is introduced in [3] and [4]. In contrast with [3, 4], the focus of this paper is a comparison between two common pricing schemes (i.e., fixed vs dynamic) for the interactions between the transmission and microgrid systems. The fixed pricing scheme for the microgrid is implemented under an operation scheme that is not included in [3, 4]. In addition, this paper analyzes the incremental benefit of utilizing the bi-level framework as the number of distribution systems modeled increases, to explore the impact of gradual system changes on operations, and potential challenges under the current (single-level) optimization framework. Finally, this paper analyzes the causes of incremental system cost changes as the number of microgrids increases. These simulations include data for the distributed generation and microgrid demand response under alternative system configurations; an analysis distinct from that presented in [3, 4].

The bi-level framework developed here is consistent with standard Stackelberg game theory used to model interactions between two economic players (i.e., leader and follower) $[5,6,7]$. Specifically, the leader makes the first move with some expectation for the follower's move. The follower then reacts to the leader's move optimally. The co-opimization modeling framework for the two power system levels, representing the two physical voltage levels in the power system, is discussed below.

The integrated system of the transmission system and microgrids can operate either in islanded or cooperative modes. In islanded mode, the microgrids do not interact with the bulk power system and so do not exchange energy with the transmission system. In the cooperative mode, the two systems are co-optimized such that they do exchange energy and reserves services, to the extent that these are part of an optimal, least cost, solution. The transmission system optimization phase determines the locational marginal pricing (LMP) to be used as the price of microgrid energy import and export as well as the price for the microgrid DR purchases. The microgrid responds to the price by determining the amount of energy exchange and provision of DR to the transmission system. This exchange of energy provides feedback to the transmission system level, and affects the determination of the LMP.

\subsection{Upper Level: Transmission System Unit Commitment}

For the upper level transmission system unit commitment decision, central station generation and aggregated low-voltage system loads are connected to generation and load buses, respectively. In this framework, microgrids are modeled as detailed networks with distributed energy resources (generation, responsive demand and storage), connected to the high voltage grid at specified buses. This upper level problem solves the day-ahead unit commitment for both energy and reserves. The objective function represents costs for all energy resources, central stations, renewable generation and distributed resources, and is minimized while accounting for uncertainties. The transmission system will either use power from central generating units or power exported from microgrids. In the reserves market, the objective is to minimize the cost of reserves while accounting for renewable energy (specifically wind energy) uncertainty. Reserves can be provided from transmission system generating resources as well as from low-voltage system demand response. Energy and ancillary services are co-optimized for the unit commitment problem [8].

A compact formulation for the upper-level level problem is

$$
\begin{aligned}
& \min _{x \in X} F(x, y) \\
\text { s.t.: } & G_{i}(x, y) \leq 0, i \in\{1,2, \ldots, I\} \\
& H_{k}(x, y)=0, k \in\{1,2, \ldots, K\}
\end{aligned}
$$

In (1), the variable set of the upper-level problem is $X$, the objective function is $F(x, y)$, and the functions $G_{i}, i=1, \ldots, I$ and $H_{k}, k=1, \ldots, K$, are the left-hand sides of the constraints. The co-operative 


$$
x_{t}=\left[w_{g, t}, p_{g, t}, r_{g, t}^{u p}, r_{g, t}^{d n}, p_{t}^{d r}, c_{t}^{i m}, c_{t}^{e x}\right]
$$

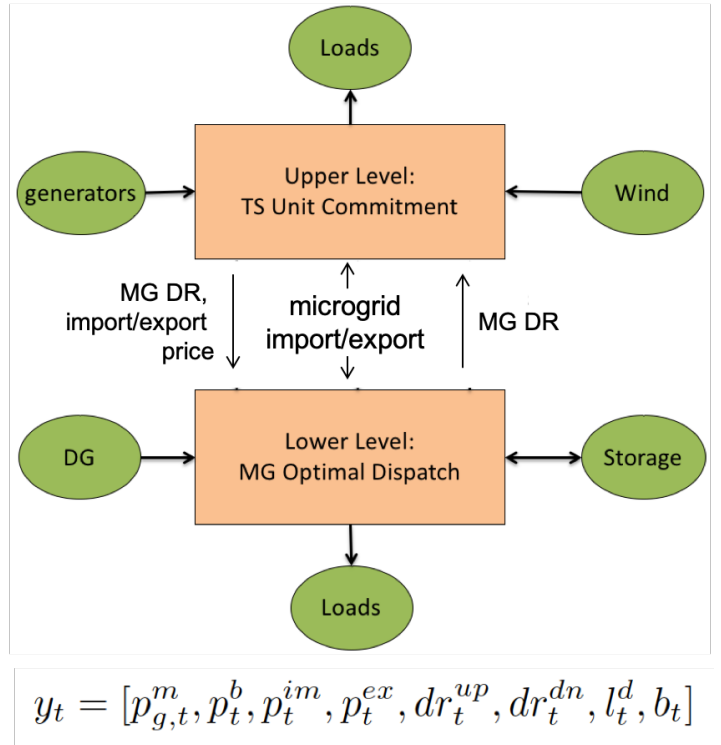

Figure 1. The upper-level transmission system determines LMP; the lower-level microgrid responds with DG \& DR dispatch and energy exchange.

behavior between the two systems is illustrated in a bi-level optimization structure in Figure 1. The optimization variables for the upper-level problem are shown by the vector $x_{t}$ :

$$
x_{t}=\left[w_{g, t}, p_{g, t}, r_{g, t}^{u p}, r_{g, t}^{d n}, p_{t}^{d r}, c_{t}^{i m}, c_{t}^{e x}\right]
$$

in which the variables are defined as: the generator commitment status, $w_{g, t}$, generator output, $p_{g, t}$, generator upward and downward reserve, $r_{g, t}^{u p}, r_{g, t}^{d n}$, microgrid demand response price, $p_{t}^{d r}$, and the prices of the microgrid imported and exported energy, $c_{t}^{i m}, c_{t}^{e x}$.

The unit commitment formulation of the upper-level problem is based on that in [9]. The objective function for minimizing the cost of generation, reserves, energy exchange with the microgrid, and microgrid demand response, is

$$
\begin{aligned}
F\left(\left\{x_{t}\right\}_{t=1}^{T}\right) & =\sum_{t=1}^{T} \sum_{g=1}^{G}\left(C_{g}^{c} w_{g, t}+C_{g}^{1} p_{g, t}\right. \\
& +C_{g}^{2}\left(p_{g, t}\right)^{2}+C_{g}^{r}\left(r_{g, t}^{u p}+r_{g, t}^{d n}\right) \\
& \left.-c_{t}^{i m} p_{t}^{i m}+c_{t}^{e x} p_{t}^{e x}+p_{t}^{d r}\left(d r_{t}^{u p}+d r_{t}^{d n}\right)\right)
\end{aligned}
$$

and is minimized subject to the following constraints:

$$
\begin{aligned}
& \sum_{g=1}^{G} p_{g, t}-\left(\mathbf{1}_{1 \times N_{b}} \cdot L_{t}\right)+W_{t}^{f}=p_{t}^{i m}-p_{t}^{e x}, \\
& t \in\{2, \ldots, T\}, g \in\{1, \ldots, G\} \\
& -\bar{L} \leq G S F \times p_{i n j_{-} t} \leq \bar{L}, \quad t \in\{1, \ldots, T\} \\
& -\bar{L} \leq G S F \times p_{\text {inj_t }}^{*} \leq \bar{L}, \quad t \in\{1, \ldots, T\} \\
& \underline{P}_{g} \times w_{g, t} \leq p_{g, t} \leq \bar{P}_{g} \times w_{g, t} \\
& t \in\{1, \ldots, T\}, g \in\{1, \ldots, G\} \\
& \left(p_{g, t}+r_{g, t}^{u p}\right)-\left(p_{g, t-1}-r_{g, t-1}^{d n}\right) \leq \bar{R}_{g} \text {, } \\
& t \in\{2, \ldots, T\}, g \in\{1, \ldots, G\} \\
& \underline{R}_{g} \leq\left(p_{g, t}-r_{g, t}^{d n}\right)-\left(p_{g, t-1}+r_{g, t-1}^{u p}\right), \\
& t \in\{2, \ldots, T\}, g \in\{1, \ldots, G\} \\
& W_{t}^{u p} \leq d r_{t}^{u p}+\sum_{g=1}^{G} r_{g, t}^{d n}, \quad t \in\{1, \ldots, T\} \\
& W_{t}^{d n} \leq d r_{t}^{d n}+\sum_{g=1}^{G} r_{g, t}^{u p}, \quad t \in\{1, \cdots, T\}
\end{aligned}
$$

Equation (3) is the power balance with $\mathbf{1}_{1 \times N_{b}}$ an $N_{b}$-dimentional vector of $1 \mathrm{~s}$. The dot product $\mathbf{1}_{1 \times N_{b}}$. $L_{t}$ is the total load in the system. Equations (4) and (5) are power flow line limits, $p_{i n j_{-} t}$ denoting the nodal net power injection vector for generation and demand at all the buses in period $t$. Forecast errors in wind power, generation and demand response are shown in $p_{i n j_{t} t}^{*}$. Equation (6) specifies the generator minimum and maximum output, and ramping limits are in equations (7) and (8). Equations (9) and (10) ensure sufficient generator reserve and microgrid demand response to mitigate deviations from forecast wind generation. The representation of wind forecast errors is discussed below. Complete nomenclature is included in the appendix.

Wind Forecast Errors. The wind power generation is all located in windfarms, at the high-voltage system level. The wind data are from the NREL-Eastern Wind Integration Study dataset [10]. Using three years of data, 24-hour trajectories are grouped to identify a set of 54 trajectories representing possible wind realizations. The central trajectory of the group is selected as the wind power forecast, and the remaining trajectories are used to estimate the distribution of forecast errors. Based 
on the forecast error distribution, 10,000 scenarios are generated to represent an uncertainty set of wind realizations, each of which would introduce error with respect to the base case wind forecast. Those error scenarios are then added to the forecast to create wind generation scenarios [11].

Deviations from the day-ahead wind forecasts, $W_{t}^{u p}$ and $W_{t}^{d n}$, are determined as the difference between the base case generation scenarios and the realizations for period $t$. The transmission generation reserve, $r_{g, t}^{d n}$ or $r_{g, t}^{u p}$, and microgrid demand response, $d r_{t}^{u p}$ or $d r_{t}^{d n}$, are used to offset the wind power deviation from the forecast. The increase or decrease in the microgrid dispatchable load relative to the baseline $l_{t}^{d}$ is $d r_{t}^{u p}$ and $d r_{t}^{d n}$. This approach adheres to robust optimization for the bi-level framework since the reserve allocation is optimized for the worst case wind scenarios.

\subsection{Lower-Level: Microgrid Optimal Power Flow}

The microgrid model includes distributed generation (DG), energy storage, aggregated dispatchable load, and non-dispatchable load. Each microgrid has some dispatchable, or flexible load and some non-dispatchable, non-responsive load. The modeling framework is designed to allow the microgrid and the high-voltage transmission system to exchange power with bi-directional flow. For the dispatchable load, any margin between the upper or lower bounds and its dispatch point is available to provide upward or downward demand response as needed by the transmission system (particularly to balance wind uncertainties). The objective of the microgrid is to minimize the cost of meeting its load either with its own distributed generation or with energy import from the transmission system, as well as to maximize revenue from providing demand response and energy export to the transmission ssytem [12].

A general formulation for the lower-level problem is:

$$
\begin{aligned}
& \min _{y \in Y} f(x, y) \\
\text { s.t.: } & g_{j}(x, y) \leq 0, j \in\{1,2, \ldots, J\} \\
& h_{m}(x, y)=0, m \in\{1,2, \ldots, M\}
\end{aligned}
$$

In (11), the variable set of the lower-level problem is $Y$, the objective function is $f(x, y)$, and the constraints are defined by the functions $g_{j}, j=1, \ldots, j$ and $h_{m}, m=1, \ldots, M$, and their corresponding right-hand sides. In figure 1, the lower-level optimization variables are shown in the vector $y_{t}$ :

$$
y_{t}=\left[p_{t}^{m}, p_{t}^{b}, p_{t}^{i m}, p_{t}^{e x}, d r_{t}^{u p}, d r_{t}^{d n}, l_{t}^{d}, b_{t}\right]
$$

The formulation of the microgrid optimal dispatch problem is based on [13]. The objective is to determine the generation dispatch schedule, $p_{t}^{m}$, the energy storage power output, $p_{t}^{b}$ (]i.e., the energy storage charging and discharging decision), the microgrid energy import schedule, $p_{t}^{i m}$, and export schedule, $p_{t}^{e x}$, the dispatchable load profile, $l_{t}^{d}$, the upward and downward demand response, $d r_{t}^{u p}$ and $d r_{t}^{d n}$, as provided by the dispatchable load, and the energy storage energy state, $b_{t}$.

The goal is cost minimization for the microgrid, including the cost of generation, energy storage, energy exchange with the transmission system, and microgrid demand response. The dispatchable load consumption, energy export, and demand response revenue are negative costs, and are therefore maximized. The objective function is:

$$
\begin{aligned}
f\left(\left\{y_{t}\right\}_{t=1}^{T}\right) & =\sum_{t=1}^{T}\left(C^{m 1} p_{t}^{m}+C^{m 2}\left(p_{t}^{m}\right)^{2}\right. \\
& +C^{b} b_{t}+c_{t}^{i m} p_{t}^{i m}-c_{t}^{e x} p_{t}^{e x}+C^{d r 1}\left(d r_{g, t}^{u p}\right. \\
& \left.+d r_{g, t}^{d n}\right)+C^{d r 2}\left(\left(d r_{t}^{u p}\right)^{2}+\left(d r_{t}^{d n}\right)^{2}\right) \\
& \left.-C^{d} l_{t}^{d}-p_{t}^{d r}\left(d r_{t}^{u p}+d r_{t}^{d n}\right)\right)
\end{aligned}
$$

and is subject to:

$$
\begin{array}{cl}
p_{t}^{m}-p_{t}^{b}-L_{t}^{i}-l_{t}^{d}=p_{t}^{e x}-p_{t}^{i m}, \quad t \in\{1, \ldots, T\} \\
\underline{P}^{m} \leq p_{t}^{m} \leq \bar{P}^{m}, \quad t \in\{1, \ldots, T\} \\
\underline{L}_{t}^{d} \leq l_{t}^{d} \leq \bar{L}_{t}^{d}, & t \in\{1, \ldots, T\} \\
l_{t}^{d}+d r_{t}^{u p} \leq \bar{L}_{t}^{d}, & t \in\{1, \ldots, T\} \\
l_{t}^{d}-d r_{t}^{d n} \geq \underline{L}_{t}^{d}, & t \in\{1, \ldots, T\} \\
0 \leq d r_{t}^{u p} \leq W_{t}^{u p}, & t \in\{1, \ldots, T\} \\
0 \leq d r_{t}^{d n} \leq W_{t}^{d n}, & t \in\{1, \ldots, T\} \\
\underline{P}^{b} \leq p_{t}^{b} \leq \bar{P}^{b}, & t \in\{1, \ldots, T\} \\
\underline{B} \leq b_{t} \leq \bar{B}, & t \in\{1, \ldots, T\} \\
b_{t}=b_{t-1}+p_{t-1}^{b}, & t \in\{1, \ldots, T\}
\end{array}
$$




$$
\begin{array}{ll}
0 \leq p_{t}^{i m}, & t \in\{1, \ldots, T\} \\
0 \leq p_{t}^{e x}, & t \in\{1, \ldots, T\}
\end{array}
$$

Equation (13) is the power balance for the microgrid. Equation (14) limits the microgrid's generation within upper and lower bounds. Equation (15) defines the bounds on the initial dispatch level for flexible, or dispatchable load. Equations (16) and (17) limit the up or down response of the dispatchable load to lie between the upper and lower bounds for these loads. Additionally, the demand response is constrained to not exceed the wind power deviation from the forecast, as specified in equations (18) and (19). Equations (20) and (21) update the energy storage output and energy state, and ensure they remain within their bounds. The energy storage state transition dynamics are shown in equation (22). A positive or negative $p_{t-1}^{b}$ value corresponds to charging and discharging of the energy storage, respectively. Finally, inequalities (23) and (24) are non-negativity constraints on the import and export of power for the microgrid.

\subsection{Solution Approach}

Bi-level optimization problems simulate the interaction of market players with conflicting objectives and constraints. The lower-level problem in this study has the advantage of being convex and satisfies Slater's constraints qualifications. These properties allow the lower level problem to be replaced by its associated Karush-Kuhn-Tucker conditions, and combined with the upper-level problem to form a single level problem that represents the optimization of each system appropriately [14]. The bi-linearity in the resulting single-level problem is circumvented by the big-M method and strong duality theorem. References [15],[16],[17] provide the technical details of this solution approach. The resulting problem is a mixed-integer linear problem which could be solved by various solvers in the market such as Gurobi and Cplex.

\subsection{Power System Model}

The transmission model described in Section 2.1 is applied to the IEEE 30-bus system shown in Figure 2. The total generation capacity of the system is $335 \mathrm{MW}$. A wind farm is positioned at bus 5. For consistency with realistic market conditions, it is assumed that the energy buy-back price in the wholesale market is slightly lower than the energy sale price. As a result, the microgrid export cost, $c_{t}^{e x}$, is defined as $0.9 \times$ $c_{t}^{i m}$. A baseline $25 \mathrm{MW}$ microgrid with parameters provided in Table 1 is used to demonstrate system operations under this bi-level framework. The six

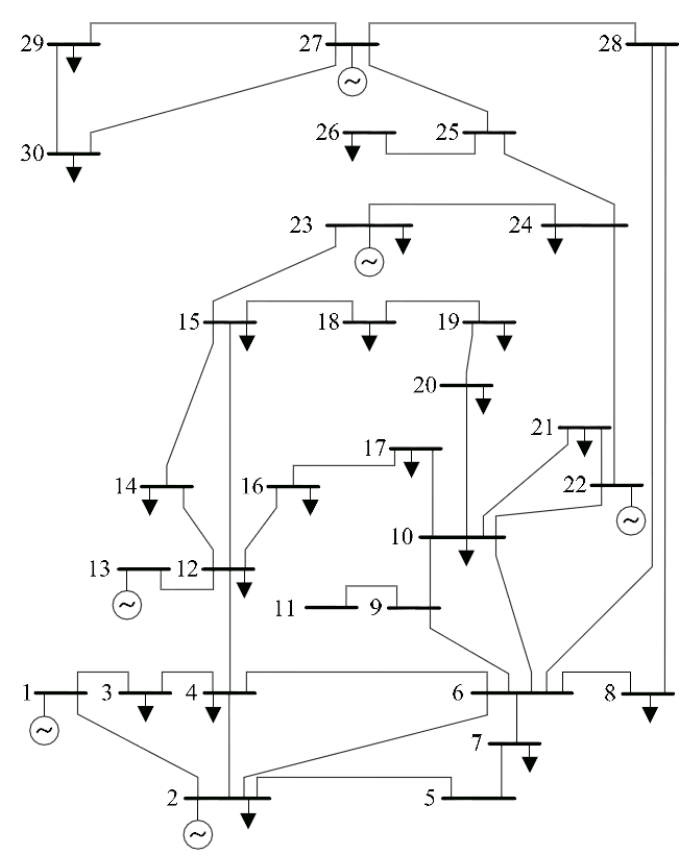

Figure 2. IEEE 30-bus system. [18]

bus diagram for the low voltage system is shown in Figure 3. The microgrid includes a generator, a storage unit, aggregated dispatchable load, and non-dispatchable load, and is able to operate in islanded and grid-connected modes.

\begin{tabular}{|ll|ll|}
\hline Parameter & Value & Parameter & Value \\
\hline$\underline{L}_{t}^{d}$ & $6.6 \mathrm{MW}$ & $C^{d r 1}$ & $\$ 0.4 / \mathrm{MW}$ \\
$\bar{L}_{t}^{d}$ & $12 \mathrm{MW}$ & $C^{d r 2}$ & $\$ 0.3 / \mathrm{MW}$ \\
$L_{t}^{i}$ & $12 \mathrm{MW}$ & $C_{t}^{d}$ & $\$ 6 / \mathrm{MW}$ \\
$\bar{B}$ & $10 \mathrm{MW}$ & $\bar{P}_{t}^{d}$ & $\$ 1 / \mathrm{MW}$ \\
$\underline{B}$ & $0 \mathrm{MW}$ & $\bar{P}^{m}$ & $25 \mathrm{MW}$ \\
$C^{b}$ & $\$ 0.1 / \mathrm{MW}$ & $\underline{P}^{m}$ & $0 \mathrm{MW}$ \\
$C_{t}^{m 1}$ & $\$ 4 / \mathrm{MW}$ & $\underline{P}^{b}$ & $-5 \mathrm{MW}$ \\
$C_{t}^{m 2}$ & $\$ 0.07 / \mathrm{MW}$ & $\bar{P}^{b}$ & $5 \mathrm{MW}$ \\
\hline
\end{tabular}

Table 1. microGrid parameter values

\section{Results}

The purpose of these simulations is two-fold. First is the investigation of the degree to which the proposed bi-level optimization framework is effective in quantifying the appropriate cost allocation between the high and low voltage systems in the interconnected electric power system. Second is the analysis of a set of possible future configurations of the power system, focusing on increasing the number of microgrids 


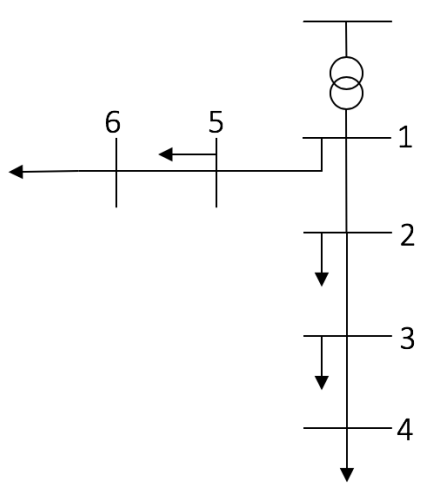

Figure 3. 6 bus low voltage system.

interconnected with the transmission system. Results from the bi-level optimization framework are compared to those from the traditional single-level optimization. The single- and bi-level formulations differ in that the single-level optimization objective function is the sum of those from the upper and lower level problems under the bi-level framework, and the constraints are the combined set of the constraints from the upper and lower level problems. The bi-level framework uses the KKT conditions to represent the leader-follower aspects of the co-optimized system, and also is capable of modeling the details of network constraints in the low voltage network. However these details are not included in the existing, traditional optimization framework. Therefore, to ensure a meaningful comparison between formulations, low-voltage network constraints are not implemented in either framework in the results presented below. This ensures that the differences in results presented here are entirely due to the differences in the problems structures.

Figures 4 and 5 show the system costs as determined by the bi-level framework compared to those determined by the standalone single-level optimization framework. The high voltage transmission system has interconnectged microgrids, modeled as active low voltage systems with distributed generation, demand response, and storage. The single-level optimization assumes islanded mode operation for the microgrids in as much as they do not actively interact with, or exchange energy bi-directionally with, the transmission system. In contrast, the bi-level co-optimization allows for bi-directional energy flow at each level. Three cases are simulated with each optimization framework, including one, three and five baseline microgrids at bus five. Since there is no congestion in the system, the locations of the microgrids are not critical to the results presented.

Results show that microgrid cost increases as

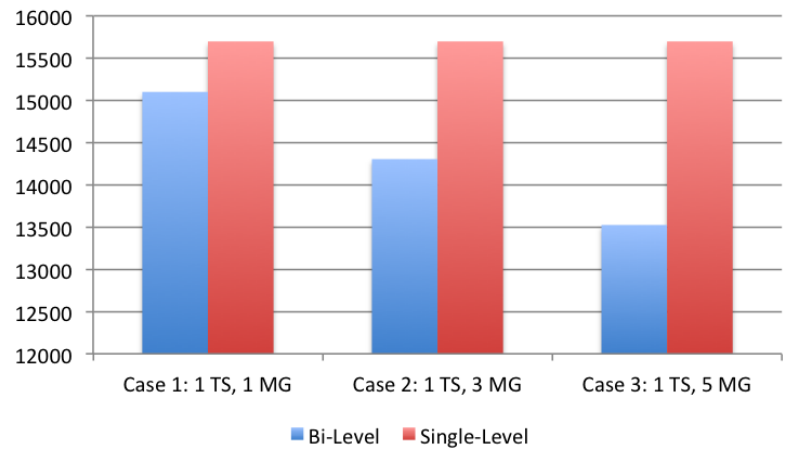

Figure 4. Comparison of Transmission System Cost between Bi-Level and Single-Level Optimization as Modeled with 1, 3 \& 5 MicroGrids

the number of microgrids in the system increases; such cost increases being balanced by transmission system cost decreases. The costs are seen to shift because the transmission system has more supply choices under the bi-level scheme with increased access to demand response resources and microgrid energy export. Access to more supply options lowers the corresponding costs in the transmission system under the co-operative framework. However, with the microgrid contributions to transmission system balancing needs being better represented, the associated microgrid costs from supplying demand response and energy exports are seen to rise.

Under the single-level framework, there are no interactions between the high- and low-voltage networks, so the microgrid costs do not change as the number of microgrids in the system increases. Figure 6 shows the changing use of DG and DR within the microgrids as the number of connected microgrids increases from one to three to five. This chart shows the average DER dispatch, demonstrating that proportionally more DER is used by the system as the number of microgrids, and associated DER capacity, increases.

Figure 7 compares costs in the high and low voltage systems under the bi-level framework versus the fixed LMP single-level scheme. Under this single-level optimization, the transmission system determines the nodal LMPs without using generation, demand response or price information from the microgrids. This transmission system LMP is used as the deterministic price for energy transactions between the microgrid and the transmission system. In this case, three microgrids are positioned at bus 5 (MG1 with base configuration), bus 10 (MG2 with 0.8 capacity of the base configuration) and bus 15 (MG3 with 1.2 capacity 


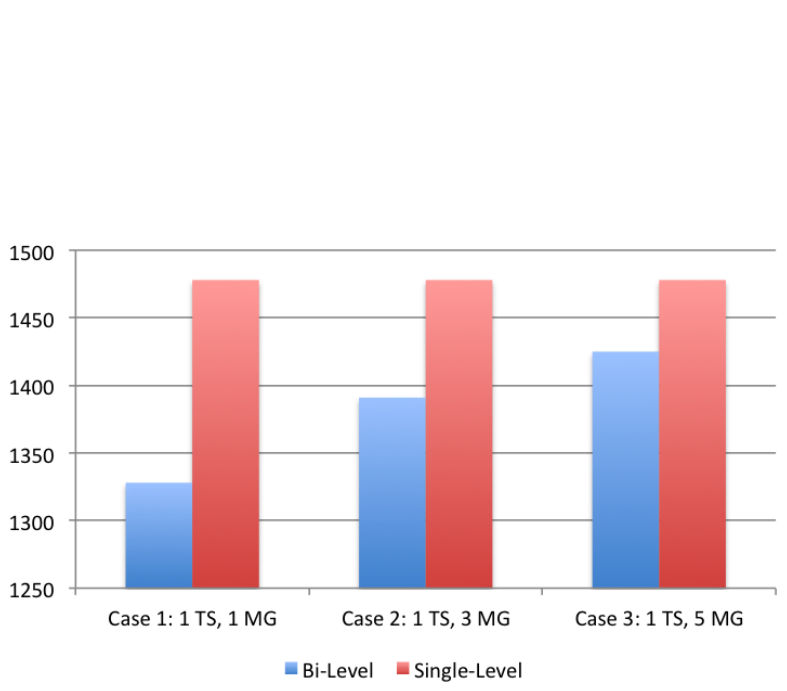

Figure 5. Comparison of Average Distribution System Cost between Bi-Level and Single-Level Optimization in System with 1, 3 \& 5 MicroGrids

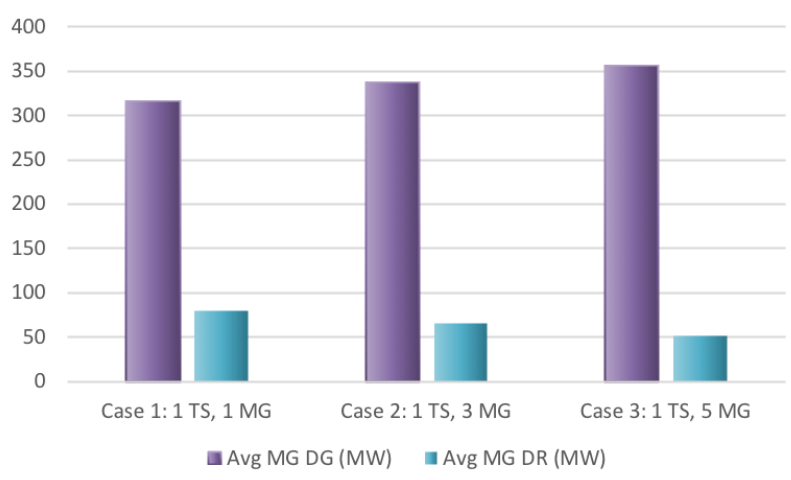

Figure 6. Average Microgrid DG and DR Dispatch with 1,3 \& 5 MicroGrids

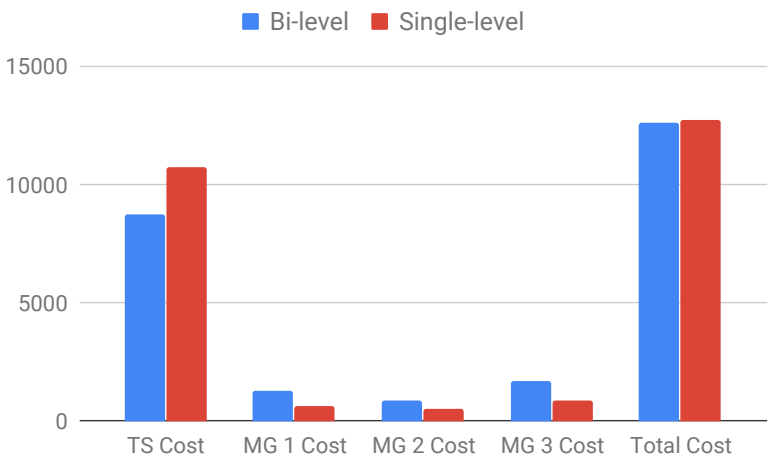

Figure 7. Transmission System \& Microgrid Costs: Comparison between Bi-Level and Single-Level Optimization

of the base configuration).

With deterministic LMP, the microgrid cost is lower in the traditional single-level optimization framework than in the bi-level framework. Microgrid costs are inappropriately attributed to the transmission system in the single-level optimization framework since detailed microgrid data and information are not taken into consideration in the determination of the pricing in this traditional framework. With the bi-level optimization, the transmission system cost is lower as the price is optimized for the interconnected system, assigning costs to the distribution system as appropriate. Under the bi-level framework, the maximum possible wind penetration, while maintaining system stability, is higher than under the single-level approach due to flexibility in pricing (with the bi-level framework). The total system operation cost under the bi-level framework is also lower.

Figures 8 and 9 show the high voltage transmission system cost with traditional, radial and passive distribution systems, again comparing results from single-level and bi-level optimization frameworks. The three distribution systems are positioned at the same buses and have the same configurations as the baseline microgrid, but now without distributed generation or energy storage. The traditional, single-level framework incurs higher average costs for the distribution systems as the number of connected distribution systems increases, due to higher generator costs from higher generation dispatch. The transmission system cost decreases accordingly.

Under the bi-level framework, the distribution system cost is lower than that under the single level counterpart as the distribution system participation is explicitly considered in the lower level objective function. These results demonstrate that the distribution 


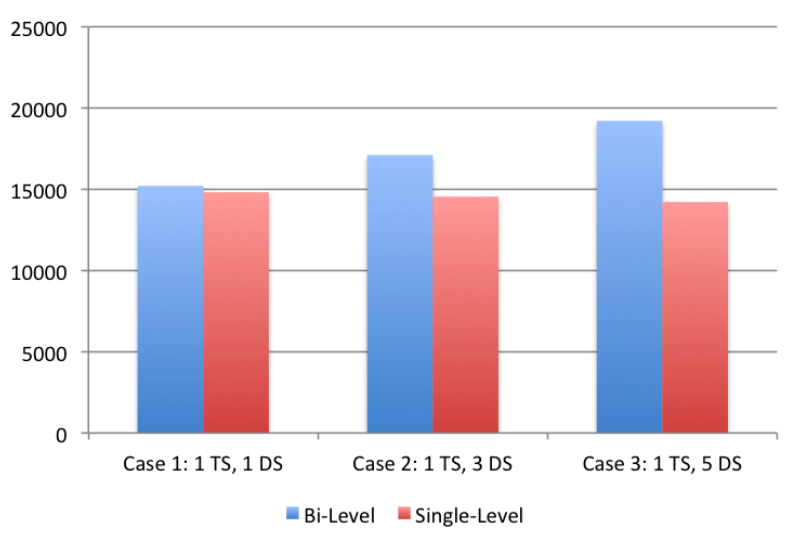

Figure 8. Comparison of Transmission System Cost between Bi-Level and Single-Level Optimization as Modeled with 1, 3 \& 5 Passive Distribution Systems

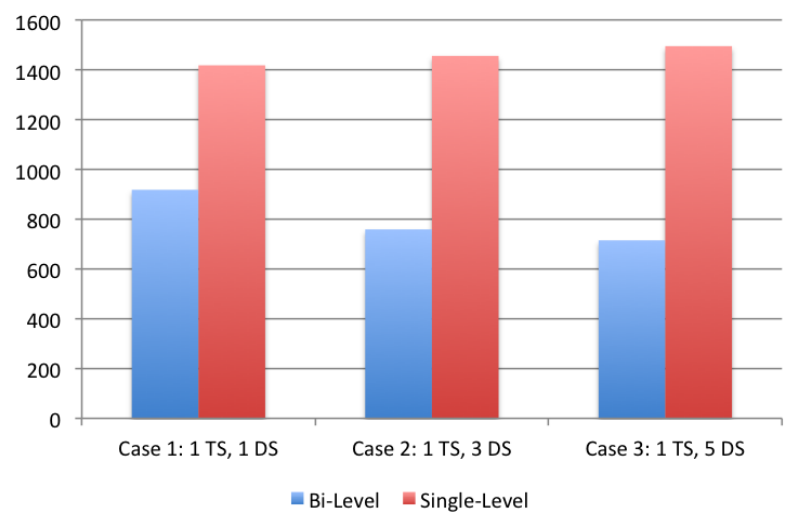

Figure 9. Comparison of Average Distribution System Cost between Bi-Level and Single-Level Optimization in System with 1, 3 \& 5 Passive Distribution Systems

system cost under the single level framework is under-evaluated; a result which will become increasingly inadequate as the distribution systems become more dynamic and multi-functional in the future power system. In addition, the decreasing average distribution system cost under the bi-level framework means that representing the distribution system as individual optimization entities yields additional benefits to each distribution system as more distribution systems participate in this optimization framework due to the mutual arbitrage among the optimization entities. The transmission system cost increases with more distribution systems participating in this bi-level framework as the transmission system has decreased advantage over the distribution system operations, as is appropriate.

\section{Conclusion}

This paper proposes the use of a bi-level optimization algorithm to replace the traditional single-level optimization currently used for the analysis of power system costs and generator dispatch. Though the existing single-level framework adequately determines system performance at low levels of DER and microgrid penetration, as the use of new technologies and active devices becomes more widespread, the traditional single-level framework will become inadequate. The paper develops a bi-level optimization framework and demonstrates its use with the IEEE 30 bus test system and an increasing number of active microgrids. The system simulations presented in this paper demonstrate that as DER use increases a bi-level optimization framework more accurately determines power system operating costs than does the traditional single-level optimization algorithm. Further detailed simulations could demonstrate additional challenges introduced by increasingly active low voltage systems, and explore the demonstrate benefits of more detailed modeling of system interaction via bi-level optimization.

\section{Appendix: Nomenclature}

Variables
$r_{g, t}^{u p} / r_{g, t}^{d n}$
$p_{t}^{d r}$
$p_{g, t}$
$p_{t}^{m}$
$b_{t}$
$p_{t}^{e x} / p_{t}^{i m}$
$c_{t}^{e x} / c_{t}^{i m}$
$p_{t}^{b}$
$l_{t}^{d}$
$d r_{t}^{u p} / d r_{t}^{d n}$
$p i n j_{t}$
$w_{g, t}$

upward/downward reserve of transmission system generator $g$ in period $t$ microgrid demand response price in period $t(\$ / \mathrm{MW})$ generation of transmission system generator $g$ in period $t$ microgrid generation in period $t$ microgrid storage energy state at the beginning of period $t$ microgrid exported/imported power in period $t$ price of microgrid exported/imported power in period $t$ microgrid storage power (charging/discharging) output in period $t$ microgrid aggregated dispatchable load in period $\mathrm{t}$ upward/downward demand response of microgrid dispatchable load in period $t$ transmission bus power injection vector in period $t$ transmission system generator commitment variable for generator $g$ in period $t$ 


\begin{tabular}{|c|c|}
\hline Paremeters & \\
\hline$T$ & length of the planning horizon \\
\hline$G$ & $\begin{array}{l}\text { total number of generators in the } \\
\text { transmission system }\end{array}$ \\
\hline $\bar{R}_{g} / \underline{R}_{g}$ & $\begin{array}{l}\text { upper/lower ramp rate limit of } \\
\text { transmission system generator } g\end{array}$ \\
\hline $\bar{P}_{g} / \underline{P}_{g}$ & $\begin{array}{l}\text { generation upper/lower bound of } \\
\text { transmission system generator } g\end{array}$ \\
\hline $\bar{L}$ & transmission system line limit \\
\hline$C_{g}^{1} / C_{g}^{2}$ & $\begin{array}{l}\text { linear/quadratic cost coefficient ( } \$ / \mathrm{MW}) \\
\text { of transmission system generator }\end{array}$ \\
\hline$C_{g}^{c}$ & $\begin{array}{l}\text { commitment cost coefficient }(\$ / \mathrm{MW}) \text { of } \\
\text { transmission system generator } g\end{array}$ \\
\hline$C_{g}^{r}$ & $\begin{array}{l}\text { reserve cost coefficient }(\$ / \mathrm{MW}) \text { of } \\
\text { transmission system generator } g\end{array}$ \\
\hline$G S F$ & $\begin{array}{l}\text { generation shift factor matrix for } \\
\text { transmission system }\end{array}$ \\
\hline$W_{t}^{f}$ & $\begin{array}{l}\text { transmission forecasted wind power } \\
\text { in period } t\end{array}$ \\
\hline$W_{t}^{u p} / W_{t}^{d n}$ & $\begin{array}{l}\text { upward/downward deviation from the } \\
\text { forecasted wind power in period } t \text { in } \\
\text { the transmission system }\end{array}$ \\
\hline$N_{b}$ & number of buses in the transmission system \\
\hline$L_{t}$ & $\begin{array}{l}\text { transmission system load power vector } \\
\text { in period } t\end{array}$ \\
\hline$\underline{L}_{t}^{d} / \bar{L}_{t}^{d}$ & $\begin{array}{l}\text { lower/upper bound for microgrid } \\
\text { aggregated dispatchable load in period } t\end{array}$ \\
\hline$L_{t}^{i}$ & microgrid inelastic load in period $t$ \\
\hline $\bar{B} / \underline{B}$ & $\begin{array}{l}\max / \text { min level of microgrid storage } \\
\text { energy state }\end{array}$ \\
\hline$C^{b}$ & $\begin{array}{l}\text { microgrid storage energy maintenance cost } \\
\text { coefficient }(\$ / \mathrm{MW})\end{array}$ \\
\hline$C^{m 1} / C^{m 2}$ & $\begin{array}{l}\text { microgrid generation linear/quadratic cost } \\
\text { coefficient }(\$ / \mathrm{MW})\end{array}$ \\
\hline$C^{d}$ & $\begin{array}{l}\text { microgrid utility for consuming } \\
\text { dispatchable load }\end{array}$ \\
\hline$C^{d r 1} / C^{d r 2}$ & $\begin{array}{l}\text { microgrid linear/quadratic demand response } \\
\text { cost coefficient }\end{array}$ \\
\hline $\bar{P}^{m} / \underline{P}^{m}$ & $\begin{array}{l}\text { upper/lower bound on microgrid } \\
\text { generation }\end{array}$ \\
\hline$\underline{P}^{b} / \overline{1}$ & $\begin{array}{l}\text { nicrogrid storage discharging/charging } \\
\text { imit }\end{array}$ \\
\hline
\end{tabular}

\section{Acknowledgments}

The authors thank C. Lindsay Anderson for her suggestions.

This material is based upon work supported in part by PSERC, project T-61, and in part by the US Department of Energy under Award Number DE-OE0000843. Disclaimer: This report was prepared as an account of work sponsored by an agency of the United States Government. Neither the United States Government nor any agency thereof, nor any of their employees, makes any warranty, express or implied, or assumes any legal liability or responsibility for the accuracy, completeness, or usefulness of any information, apparatus, product, or process disclosed, or represents that its use would not infringe privately owned rights. Reference herein to any specific commercial product, process, or service by trade name, trademark, manufacturer, or otherwise does not necessarily constitute or imply its endorsement, recommendation, or favoring by the United States Government or any agency thereof. The views and opinions of the authors expressed herein do not necessarily state or reflect those of the United States Government or any agency thereof.

\section{References}

[1] D. P. Tuttle, G. Gülen, R. Hebner, C. W. King, D. B. Spence, J. Andrade, J. A. Wible, R. Baldwick, and R. Duncan, "The history and evolution of the us electricity industry," White Paper UTEI/2016-05-2, 2016.

[2] L. Anderson, L. Zéphyr, and J. Cardell, "A vision for co-optimized t\&d system interaction with renewables and demand response," in Proceedings of the 50th Hawaii International Conference on System Sciences, 2017.

[3] J. Liu, Z. Zephyr, J. Cardell, and L. Anderson, "Co-optimization of transmission and microgrid operations: Leveraging renewable generation and demand response," Under review.

[4] J. Liu, G. Guo, and L. Anderson, "Co-optimization of transmission and distribution operations under uncertainty from renewable generation: a comparison between a bi-level framework and the traditional framework," Under review.

[5] A. Sinha, P. Malo, and K. Deb, "A review on bilevel optimization: from classical to evolutionary approaches and applications," IEEE Transactions on Evolutionary Computation, 2017.

[6] L. N. Vicente and P. H. Calamai, "Bilevel and multilevel programming: A bibliography review," Journal of Global optimization, vol. 5, no. 3, pp. 291-306, 1994.

[7] B. Colson, P. Marcotte, and G. Savard, "An overview of bilevel optimization," Annals of operations research, vol. 153, no. 1, pp. 235-256, 2007.

[8] A. Bhardwaj, V. K. Kamboj, V. K. Shukla, B. Singh, and P. Khurana, "Unit commitment in electrical power system-a literature review," in Power Engineering and Optimization Conference (PEDCO) Melaka, Malaysia, 2012 Ieee International, pp. 275-280, IEEE, 2012.

[9] A. Papavasiliou and S. S. Oren, "Multiarea stochastic unit commitment for high wind penetration in a transmission constrained network," Operations Research, vol. 61, no. 3, pp. 578-592, 2013.

[10] G. L. R. W. Energy, "Eastern wind integration and transmission study," Technical Report, National Renewable Energy Laboratory (NREL), 2010. 
[11] C. Anderson and R. Zimmerman, "Wind output forecasts and scenario analysis for stochastic multiperiod optimal power flow," PSERC Webinar, pp. 1-38, 2011.

[12] C. Chen, S. Duan, T. Cai, B. Liu, and G. Hu, "Smart energy management system for optimal microgrid economic operation," IET renewable power generation, vol. 5, no. 3, pp. 258-267, 2011.

[13] J. Liu, G. Martinez, and C. L. Anderson, "Quantifying the impact of microgrid location and behavior on transmission network congestion," in Proceedings of the 2016 Winter Simulation Conference, pp. 1745-1756, IEEE Press, 2016.

[14] J. J. Ye, "Constraint qualifications and kkt conditions for bilevel programming problems," Mathematics of Operations Research, vol. 31, no. 4, pp. 811-824, 2006.

[15] S. Dempe and A. B. Zemkoho, "The bilevel programming problem: reformulations, constraint qualifications and optimality conditions," Mathematical Programming, vol. 138, no. 1-2, pp. 447-473, 2013.

[16] T. Ding, R. Bo, W. Gu, and H. Sun, "Big-m based miqp method for economic dispatch with disjoint prohibited zones," IEEE Transactions on power systems, vol. 29 , no. 2, pp. 976-977, 2013.

[17] W. S. Dorn, "Duality in quadratic programming," Quarterly of Applied Mathematics, vol. 18, no. 2, pp. 155-162, 1960.

[18] R. D. Zimmerman, C. E. Murillo-Sánchez, and R. J. Thomas, "Matpower's extensible optimal power flow architecture," in Power \& Energy Society General Meeting, 2009. PES'09. IEEE, pp. 1-7, IEEE, 2009. 